\title{
Erinnerungen an einen Pionier der Herzchirurgie
}

\section{Ruth Gattiker}

Prof. Dr. med., Chirurgin, Davos-Dorf

Sowohl in Fachzeitschriften [1] als auch in einem Beitrag von Stephanos Geroulanos in der Neuen Zürcher Zeitung aus dem Jahr 2000 und anlässlich des 50-jährigen Jubiläums der Herzchirurgie in Zürich wurde ausgiebig über die chirurgischen Pionierleistungen des genialen hochbegabten Chirurgen Åke Senning berichtet. Ich werde deshalb hier vermehrt meine persönliche Erinnerung an den Menschen Senning in den Vordergrund stellen.

1961 wurde Prof. Åke Senning auf Rat der chirurgischen Fakultät der Universität Zürich vom Regierungsrat als Ordinarius für Herz- und grosse Gefässchirurgie, Thorax- und Viszeralchirurgie an die neugeschaffene Chirurgische Klinik A gewählt. Zugleich wurde die Abteilung Anästhesiologie unter der Leitung von PD Dr. Georg Hossli verselbständigt.

\section{Eine Frau lernt beim Chef- Anästhesiologen in Stockholm}

1966 schrieb ein amerikanischer Chirurg: "[...] there is no field in which more cooperation between the surgeon and the anesthesiologist has been developed of necessity than that of cardiac surgery [...]». Dies wusste Senning schon 1961 und deshalb verlangte er, dass der für die Herzchirurgie in Zürich bestimmte Anästhesist sofort während drei Monaten sich bei seinem Chef-Anästhesiologen in Stockholm über die Besonderheiten dieser

\section{Seine Kritik konnte verletzend sein}

im Moment, aber nie nachtragend.

Anästhesie instruieren lassen müsse. Da ich mich für dieses Gebiet sehr interessierte, wurde ich von Hossli dazu vorgeschlagen: «Eine Frau, nein das geht nicht das gibt es nirgends ...» war die Antwort Sennings, aber es gab niemand anderes, und so flog ich zum Jahreswechsel 1960/61 sozusagen «in die Höhle des Löwen» nach Stockholm. Als ich in der Thoraxklinik des Karolinska Hospitals Senning zum ersten Mal gegenüber sass und er mich über seine Brillengläser hinausschauend kritisch musterte und zu fragen begann: Can you ..., do you know ... etc., etc., antwortete ich stereotyp

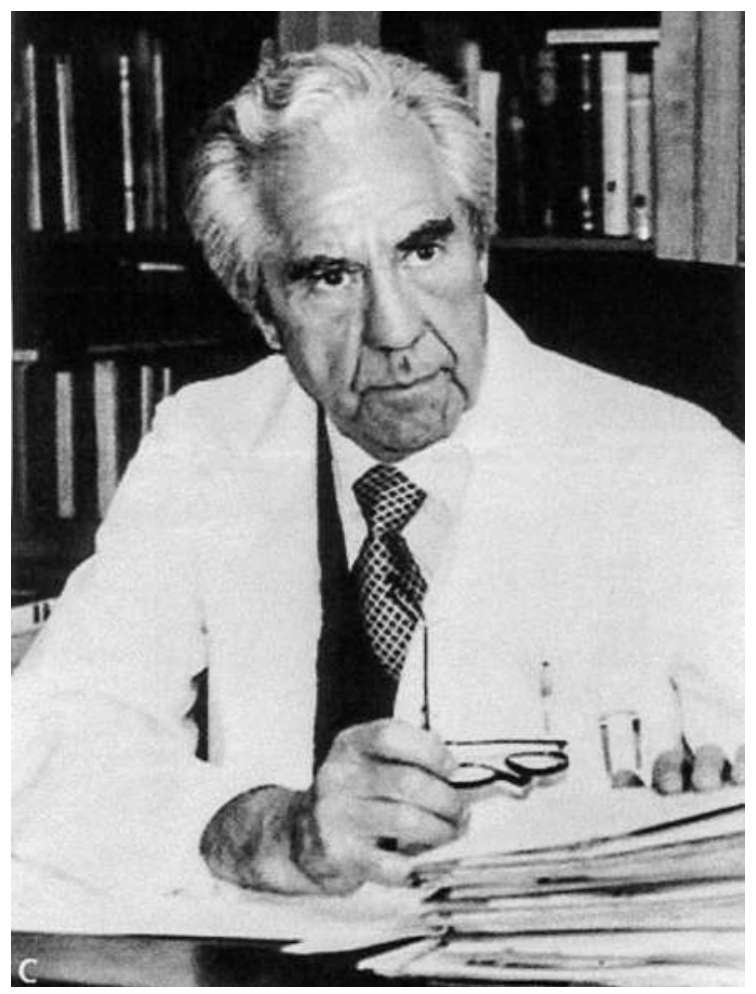

Åke Senning, Pionier der Herzchirurgie, wäre im Jahr 2015 hundert Jahre alt geworden.

mit Yes, I can, yes, I know ..., denn wie hätte ich anders können, ohne das Retourbillett in der Tasche zu gewärtigen. Das dies der Beginn einer 25-jährigen engagierten und geglückten Zusammenarbeit mit einem der renommiertesten Herzchirurgen jener Zeit werden sollte, wagte ich damals nicht zu träumen.

Mein Stockholmer Lehrer, PD Dr. Olof Norlander, war ein ausgezeichneter Vertreter seines Fachs und Kenner der verschiedenen Operationen am offenen Herzen sowie deren Ansprüche an die Anästhesie. Prof. Senning, Extraordinarius und Leiter des Experimentallabors, kam nur in den Operationssaal, wenn es galt, mit Prof. Crafoord, seinem Chef, gemeinsam zu operieren. Sie waren ein eingespieltes Team, obwohl zwischen ihnen gelegentlich ein rauer Ton herrschte. Norlander übersetzte mir zuweilen, was sie sich gegenseitig (auf Schwedisch natürlich) vorwarfen ... 
Ich wurde als vollzeitliche Mitarbeiterin inklusive Nachtdienstverpflichtung angestellt.

Senning schätzte es sehr, dass ich auch bereit war, gelegentlich seine Versuchstiere im Labor zu narkotisieren. Für koronarchirurgische Studien waren es Schweine, denn sie haben, im Gegensatz zum Hund, dieselbe Anordnung der Herzkranzgefässe wie der Mensch. Schweine sind schwierig zu intubieren und heikel zu narkotisieren. Sie sind intelligent, sehr furchtsam, stressanfällig und geraten leicht in einen Kreislaufschock, wenn man nicht sehr behutsam mit ihnen umgeht.

\section{Einander besser verstehen - dank Schlagfertigkeit}

Mitte April 1961 trat Prof. Åke Senning seine Stelle in Zürich an, anfänglich nicht zur Begeisterung seiner chirurgischen Mitarbeiter und des Operationspersonals. Seine mir bereits von Stockholm her bekannte direkte und «kein Blatt vor den Mund nehmende» Art stiess nicht auf Gegenliebe. Seine Kritik konnte verletzend sein im Moment, aber nie nachtragend. «Der behandelt uns, als wären wir der schwedische Congo" (d.h. ein unterentwickeltes Land), hörte man zuweilen munkeln. Doch als einige den Mut fanden, dem Chef zu widersprechen, und es gar auf eine witzige Art taten, gefiel ihm das und er konnte in ein befreiendes Lachen ausbrechen - und die Situation war gerettet. Auch ich stritt zuweilen mit ihm. Als er mir das Du antrug, meinte ich zögernd: «Aber wir streiten doch so oft.» «Das können wir doch per Du noch viel besser tun", meinte er.

Senning war kein konsequenter Theoretiker. Meinte man, sich nun auf etwas, das er verlangte, verlassen zu können, hatte er bereits wieder eine andere, noch bessere Variante im Kopf.

\section{Weiter denken, besser werden}

Er schätzte es aber, wenn man sich auch selber dazu anregen liess, weiter zu denken und bessere Lösungen zu finden. Ein Streitpunkt zwischen ihm und den Anästhesisten (ich hatte unterdessen drei Oberärzte und wechselnde Assistenten in meinem Team) war die Oberflächenanästhesie, die er zu Kreislaufunterbrüchen für kurzdauernde Korrekturen, wie einfache Vorhofseptum-Defekte, der Herzlungenmaschine vorzog, weil sie billiger war, wie er sagte.

Es waren aber die Anästhesisten, welche die Hypothermie und die postoperative Wiedererwärmung des
Patienten in Narkose durchführen mussten. Senning meinte, man könne doch die Patienten einfach nur noch in einer oberflächlichen Anästhesie belassen, so dass sie sich durch Shivern (Kältezittern) selber aufwärmten, das ginge viel schneller, was wir jedoch zu belastend für die Patienten empfanden.

Wenn zuweilen bei Herzoperationen festgestellt werden musste, dass die präoperative Diagnose nicht ganz stimmte, was bei angeborenen Herzfehlern zuweilen der Fall war, hielt Prof. Senning kurz inne und überlegte: In seinem Gehirn musste die ganze Hämodynamik samt Druckverhältnissen abgebildet sein, denn blitzschnell begriff er, was durch den zusätzlichen oder andersartigen Fehler verändert wurde und wie der Operationsplan angepasst werden musste. Solches mitzuerleben faszinierte mich immer wieder.

Nach den beiden Herztransplantationen vom 14. April und vom 7. Juli 1969, die beide Empfänger nur drei Monate überlebten, fasste Senning den weisen Entschluss, dieses Prozedere nicht mehr weiter zu verfolgen: Nun sind die Immunologen gefordert, sie sollen schauen, wie die Abstossung des Fremdorgans zu bekämpfen sei.

\section{Es gab immer etwas zu feiern - trotz 60-70-Stunden-Wochen.}

Åke Senning war ein kommunikativer, gemütvoller Mensch und oft zu Festen aufgelegt. Es gab - trotz der grossen Arbeitsbelastung mit 60-70-Stunden-Wochen - immer wieder etwas zu feiern: runde Geburtstage, Beförderungen, Abschiede etc. Schon kurz nach seinem Arbeitsbeginn in Zürich hat er die ganze Klinik zu sich heim eingeladen, und es wurde nicht nur gegessen und getrunken, sondern sogar getanzt. Auch später waren wir oft in seinem schönen Haus an der Belsitostrasse zu Gast und wurden von ihm und seiner Frau Ulla festlich bewirtet. Ich erinnere mich auch an ein vergnügliches Ski-Wochenende in Davos, einen gemeinsamen Klinikausflug nach München, eine Kongressreise nach Valencia und vieles mehr. Immer wusste Åke Senning das Nützliche mit dem Angenehmen zu verbinden.

Die 25-jährige Zusammenarbeit mit Prof. Åke Senning und besonders auch mit seinem Kardiologen Prof. Martin Rothlin (der leider auch verstorben ist), mit dem zusammen ich die Patienten postoperativ betreute, kann ich als die schönste Zeit meines beruflichen Lebens bezeichnen.

\footnotetext{
Referenzen

1 Largiadèr F. Professor Åke Senning 1915-2000. Schweiz Ärztezeitung 2000;81(36):2030-1.
}

In den Büelen 14

CH-7260 Davos-Dorf

rgattiker[at]bluewin.ch 